\title{
Comparative Study Between Intramedulary Interlock Nailing and Plating in Distal Metaphyseal Fractures of Tibia
}

\author{
Ravindra Hattarki, Sahil Bhagat*, Keyur Patel, Bajrang Singh, Dhanish Mehendiratta and Raghvendra Pote
}

Department of Orthopedics, D Y Patil Hospital, India

\begin{abstract}
Distal metaphyseal tibial fractures are unique. Fractures of distal tibia metaphysis occur typically as a result of axial and rotational forces on lower extremity and represents approximately $10 \%$ of the fractures of distal end of tibia.
\end{abstract}

Objective: The present study aims to compare the functional outcomes of distal metaphyseal fractures of tibia with plating to that with reamed intramedullary nailing and complication arising with different modalities of fixation.

Methodology:The study consist of 40 patients of distal metaphysical fracture of tibia consecutively treated in the period between Dec 2013 and Dec 2015 with either reamed intramedullary nailing or locked plating with open reduction method or minimally invasive techniques.

Results: Mean age of patients was 41.3 years (range, 22-65) in group 1 and 39.45 years (range, 28-56) in group $2(p=0.558)$. The average time before union was 23.3 weeks (range, 16-36 weeks) in group 1 and 25.55 weeks (range,14-41 weeks) in group 2, ( $p=0.299)$.

Conclusion: Intramedullary nail being load sharing device, comparatively early mobilization can be started. Prolonged duration of protected weight bearing was required in patients treated with locked plate and screws.

Keywords: Plating; Nailing; Distal tibia metaphyseal fractures

\section{Introduction}

Distal metaphyseal tibial fractures are unique. Fractures of distal tibia metaphysis occur typically as a result of axial and rotational forces on lower extremity and represents approximately $10 \%$ of the fractures of distal end of tibia [1,2]. The location of fracture is close to ankle joint and is not uncommon for the fracture line to extend into the joint. Distal third of the tibia has less muscle coverage in comparison to the rest of tibia. Often this fracture are communited and unstable. They can be associated with severe open or closed soft tissue injury. The degree of associated soft tissue injury is higher in distal metaphyseal fractures than with shaft fractures [3]. The management of these distal injuries is often more complex than the treatment of tibial diaphyseal fractures, leading to potential for postoperative complications and poor outcome $[4,5]$.

The goal of management of a distal metaphyseal tibial fracture is to provide stable fixation with minimal additional soft tissue injury. In last 20 years intramedullary fixation has become the mainstay of treating tibial shaft fractures by indirect reduction in closed manner. Because of its success, the indications have been extended to those of proximal and distal metaphyseal region [6]. Fixation by intramedullary device is familiar to most of the surgeons. It spares the extraosseous blood supply, allows load sharing, and avoids extensive soft tissue dissection.

Intramedullary nailing of distal metaphyseal fractures of tibia often presents the surgeon with technical difficulties due to anatomy of distal metaphyseal flare, proximity of the fracture to the ankle joint , and distal nail fixation. The medullary canal is hourglass shaped, such that tight fit of the nail is only present only in mid-diaphysis and not in distal segment.

Unlike in shaft fractures, nail insertion in distal metaphyseal fractures does not result in fracture reduction. Towards distal metaphysis, tibial cortex becomes thinner and is centrally replaced by metaphyseal secondary spongiosa and cancellous bone. In younger patients, this cancellous bone is usually dense, providing good purchase locking screws. With age the microstructure changes, resulting in an increased canal diameter and decreased bone density further lessens the nail cortex contact and screw purchase [7].

Proximity to ankle joint amplifies the bending moment of the distal segment and may facilitate fracture progression into the ankle joint [8]. But with the advent of newer nail designs with very distal cross locks and orthogonal cross locking screws, including two lateral and one anteroposterior screws help in distal fixation [9]. In addition, close attention to nailing technique including accurate guide wire placement in the Centre of the plafond and close scrutiny of the ankle joint for intra-articular fracture lines may help prevent previous problems seen with intramedullary nailing of distal metaphyseal fractures.

Since the late 1950s, open reduction and internal fixation is used for the management of distal tibia fractures. Open reduction involves extensive dissection and tissue devitalisation, creating an environment less favorable for fracture union and more prone to bone infection. Recent cadaveric studies have suggested that the circulation of distal tibia is mainly from an anastomotic network of arteries from the anterior and posterior tibial arteries, which enters tibia from medial surface. As a result less invasive methods were developed to treat metaphyseal fractures of tibia which include intramedullary nailing. Open plating of the medial aspect of distal tibia caused a statistically significant greater disruption of extraosseous blood supply of the metaphyseal area than percutaneous applied plates [10]. To improve on the poor results associated with open reduction and internal fixation, minimally invasive plate osteosynthesis techniques have been developed.

*Corresponding author: Sahil Bhagat, Mbbs, Junior Resident, D Y Patil Hospital, Kolhapur, Maharastra, India, Tel: 7507174868; E-mail: sahil.bhagat42@yahoo.com

Received May 02, 2016; Accepted May 09, 2016; Published May 18, 2016

Citation: Hattarki R, Bhagat S, Patel K, Singh B, Mehendiratta D, et al. (2016) Comparative Study Between Intramedulary Interlock Nailing and Plating in Distal Metaphyseal Fractures of Tibia. Surgery Curr Res 6: 267. doi:10.4172/21611076.1000267

Copyright: ( $) 2016$ Hattarki R, et al. This is an open-access article distributed under the terms of the Creative Commons Attribution License, which permits unrestricted use, distribution, and reproduction in any medium, provided the original author and source are credited. 
The present study aims to compare the functional outcomes of distal metaphyseal fractures of tibia managed with plating to that with reamed intramedullary nailing complication rates arising with different modalities of fixation.

\section{Material and Methods}

The study consist of 40 patients of distal metaphyseal fracture of tibia consecutively treated in the period between Dec 2013 and Dec 2015 with either reamed intramedullary nailing or locked plating with open reduction method or minimally invasive techniques. Biplanar injury radiography was evaluated to determine the fracture location and involvement of distal part of tibia. Inclusion criterias are A. Skeletally mature patient, B. Fracture involving distal $5 \mathrm{~cm}$ of tibia (AO types $\mathrm{A} 1$, A2, A3 and B1) C. All closed and Gustilo-Anderson type 1 fractures.

Exclusion criteria are A. Skeletally immature patient, B. Fractures with intra-articular extension (AO type B, C2, C3) C. Open fractures of Gustilo-Anderson type $2 \& 3$ D. All cases with DM E. Cases with psychiatric illness and moribund condition (noncompliance).

There are 2 groups of patients; group 1 included patients in which distal tibia interlock nailing was done and group 2 included patients in which distal tibia plating was done. Both groups were matched for age, sex, fracture type and comorbidities. Included patients age groups were from 18-70. Gustilo-Anderson classification was used to evaluate severity of open fractures [11].

Type 1: clean wound of less than $1 \mathrm{~cm}$ in length with minimal soft tissue injury or contamination.

Type 2: wound larger than $1 \mathrm{~cm}$ and less than $10 \mathrm{~cm}$ with moderate soft tissue damage and contamination.

Type 3: wound larger than $10 \mathrm{~cm}$ size with extensive soft tissue damage and contamination.

In majority of cases, surgery was performed following period of temporary fracture stabilization with above knee posterior plaster splint, above knee cast with window dressing to allow for soft tissue healing. All patients with compound injuries were managed on emergency basis in operating room with irrigation and debridement with subsequent plastic cover when appropriate. Intravenous antibiotics were given in compound injuries as per hospital policy.

All patients were operated after soft tissue healing was achieved, within a period of 2 to 10 days after admission in hospital. Surgery was done on standard operating table in supine position with the help of image intensifier. For intramedullary fixation we have used tibia interlocking nail with 2 proximal and 3 distal holes for locking screws, which were located in distal 3 to $4 \mathrm{cms}$ of nail to secure at least four cortices of distal tibial fragment. Patellar tendon splitting approach was used in all patients. Two hole in coronal plane and one in sagittal plane for distal fragment. Reamed intramedullary nailing was done through patellar tendon splitting approach, at least two and if possible three distal locking screws were inserted. All nails were statically locked.

For plating we have used precontoured, locked distal tibia plates. All plating cases were done through distal medial approach, either minimally invasive technique or open reduction method. Plate size was selected based on fracture anatomy pattern. Locked compression plate was inserted subcutaneously, but extreperiosteally through short longitudinal insertion over medial malleolus. In general locking screws were used in juxtaarticular segment was selected according to bone quality. Mechanically locked plate construct does not require friction fit of the plate against bone and a gap beneath the plate has advantages in preserving periosteal circulation. The locked compression plate was used as bridging construct across the diaphyseal-metaphyseal fracture. Interfragmentary compression was accomplished with either a plate independent lag screw or on selected cases through the plate.

The fibula was fixed in all cases necessary to restore the stability and normal anatomy of ankle joint, or where it was considered helpful to have a template for length. Image intensification was used intraoperatively for assessment of fracture reduction and fixation. Wound closure was achieved with absorbable suture material for the deep layers and skin clips or nonabsorbable suture to the skin. Immediate postoperative radiograph was done, limb kept in above knee slab with strict limb elevation. Patients with menial malleolar fractures were fixed with cancellous screws after intramedullary nailing. The Surgical technique can be discussed under following processes.

\section{For intramedullary nailing}

Patient positioning: Include following steps

- Place the patient supine on standard operating table after appropriate anesthesia.

- Apply the tourniquet on upper thigh.

- Confirm that unhindered anteroposterior and lateral view can be obtained under fluoroscopy.

Incision: Include following steps

- Incision is taken after limb is exsanguinated and tourniquet inflated.

- We have used patellar tendon splitting approach

- Mark the incision from lower pole of patella to the tibial tuberosity and incise the skin and subcutaneous tissue.

- Split the patellar tendon with the help of stab knife,deep to it lies the anterior tibial surface.

Entry point: Include following steps

- Entry into medullary canal is made with curved awl.

- Obtain a true anteroposterior view of upper tibia when assessing placement of awl.

- The safe zone (McConnell et al.) of entry point placement is medial to lateral tibial spine on anteroposterior view and immediately adjacent and anterior to articular surface on lateral view.

- Place the curved awl on predetermined entry site.

- Direct the awl nearly perpendicular to the shaft when it first penetrates the cortex, but gradually bring it down to a position more parallel to shaft as it is inserted more deeply to avoid violation of posterior cortex.

- Make entry wider enough to permit placement of nail without resistance.

Nail insertion: Include following steps

- Exchange the ball tip guide wire before inserting nail.

- Attach the insertion device and proximal locking screw guide to the measured size nail.

- Direct the apex of proximal bend in the nail posteriorly and mount the drill guide to direct screws from medial to lateral.

- Insert the nail with knee in flexion to avoid impingment on patella.

- Evaluate the rotational alignment by aligning iliac crest, patella and second ray of foot.

- Moderate manual pressure with gentle back and forth twisting 
motion usually is sufficient for nail insertion.

- If mallet is used, the nail should advance with each blow of nail. If nail does not advance, withdraw the nail, and perform further reaming or insert smaller diameter nail.

- When the nail has passed well into the distal fragment, remove the guide wire, to avoid incarceration, and during final seating of nail, release manual traction to allow final seating of nail.

- When the nail is fully seated, the proximal end should lie approximately 0.5 to $1 \mathrm{~cm}$ below the cortical opening of entry portal.

- The distal tip of nail should lie approximately 0.5 to $2 \mathrm{~cm}$ from subchondral bone.

- Confirm the nail position in anteroposterior and lateral views.

- Insertion of locking screws.

- Insert the proximal locking screws using the jig attached to nail insertion device.

- Place the drill sleeve through a small incision down to the bone, drilling is done, screw length is measured with help of depth gauge, and screw is inserted.

- The screw should protrude $5 \mathrm{~mm}$ beyond the far cortex to enable the screw to be removal more easily if breakage occurs.

- Use 2 proximal locking screws in most fractures.

- Tighten all connections between the insertion device, drill guide, and nail before screw insertion.

- Perform distal locking by freehand technique after "perfect circles" are obtained by fluoroscopy.

- In the lateral position, adjust the fluoroscopic beam until it is directed straight through the distal screw holes.

- Drill the hole, confirm position on anteroposterior and lateral views, measure the length and insert the screw

- At least 2 distal screws should be inserted.

Role of blocking screws: Includes following steps

- Malalignment can be prevented by using blocking screws (Krettek et al. [12]).

- $\quad 3.5$ or $4.5 \mathrm{~mm}$ screws are placed just off the midline opposite the apex of deformity.

- They are placed in anteroposterior direction, occupy the medullary cavity space and directs the guide wire and nail placement in centre of distal fragment.

\section{For open reduction \& internal fixation with plating}

Position: Include following steps

- Place the patient supine on operating table with tourniquet in place.

- Confirm both anteroposterior and lateral views on image intensifier.

- Place a sandbag under ipsilateral buttock to correct natural external rotation of the limb.

- Exsanguinate the limb by soft rubber bandage and inflate the tourniquet.

Incision: Include following steps

- Take an incision starting just distal to medial malleolus, extending the incision proximally overlying the subcutaneous border of tibia, halfway between anterior and posterior borders well proximal to fracture site.
- Deepen the skin incision to expose the periosteum overlying the tibia.

- Because the periosteum of the tibia supplies significant amount of blood to the bone it should not be removed and plate should be applied epi-periosteal position.

- Expose the fracture site and clean fracture edges with curette.

Fracture reduction and provisional fixation: Include following steps

- Position the plate on the bone and reduce the fracture manually.

- Confirm the coronal and sagittal alignment as well as plate position on the shaft.

- Fix the plate to the diaphysis with two diaphyseal provisional fixation pins allowing adequate spread between them.

- Place the metaphyseal provisional fixation pin through one of the distal holes above the joint.

- Once accurate position of the plate has been achieved, insert a conventional screw in one of the most distal plate holes to approximate the plate close to the bone.

Final plate fixation: Include following steps

- Proceed with the definitive fixation of fracture using locked screws. Usually, the metaphysis requires more screws (3-5) than the diaphysis (2-3)

- If non-locking screws are needed for either fragment, they must be inserted prior to the insertion of locking screw in that fragment.

- Confirm the placement by obtaining anteroposterior and lateral fluoroscopic images.

- Tourniquet deflated, hemostasis achieved, wound wash given and wound closure done.

Position: Include following steps

- Place the patient supine on standard operating table.

- Confirm that unhindered anteroposterior and lateral views under fluoroscopy can be achieved.

- Obtain gross alignment using manual traction or skeletal distractor.

Incision: Include following steps

- Short incision of 3 to $4 \mathrm{~cm}$ size is taken centered over medial malleolus.

- Periosteal elevator is inserted and skin tunnel is made well above the fracture site.

Plate selection: Include following steps

- Determine the appropriate length of the plate for fixation.

- In general, a longer plate allows for better mechanical advantage over a shorter plate.

- An allowance for four holes above the most proximal aspect of the fracture is recommended when selecting plate length.(AO MANUAL)

\section{Reduction and provisional fixation: Include following steps}

- It is important that fracture reduction be obtained prior to placement of locking screws.

- Temporary secure fracture fragments by using k-wires and/or reduction forceps.

- Place provisional and/or definitive fixation screws outside the 
plate if necessary

Plate positioning: Include following steps

- Insert the plate using percutaneous insertion through the distal incision for a minimally invasive procedure.

- Position the plate and reduce the fracture manually.

- Confirm coronal and sagittal alignment as well as plate position on the shaft.

- Fix the plate to the diaphysis with two diaphyseal provisional fixation pins allowing adequate spread between them.

- Place the metaphyseal provisional fixation pin through one of the distal holes above the joint.

Screw insertion: Include following steps

- Proceed with the definitive fixation of fracture using appropriate screw selections.

- If non-locking screw are needed for either fragment, they must be inserted prior the insertion of locking screws in that fragment.

- Locking screws should be used through at least two of distal holes and three proximal holes.

- Confirm screw placement by obtaining anteroposterior and lateral fluoroscopic images and close the wound over drain.

\section{Post-operative management and follow-up evaluation}

Immediate post-operative radiograph done, limb was kept in above knee plaster slab after surgery in all patients. Patients were reviewed on $2^{\text {nd }}$ and $7^{\text {th }}$ post-operative days for wound inspection. Suture removal was done on $11^{\text {th }}$ post-operative day. Patient was mobilized day after surgery with non-weight bearing crutch walking.

Intravenous antibiotics were given for 48 hours for closed fractures and for open fractures duration of intravenous antibiotics was decided depending on the condition of the wound. After the swelling subsided, immediate ankle joint mobilization was encouraged in all patients as tolerated. Decisions regarding weight bearing were made on individual basis, depending on the progress of bony union, weight of the patient and fracture characteristics. Any post-operative complication had been identified and treated appropriately. After first follow-up visit at 6 weeks, patients were only allowed to bear partial weight with crutches which could then gradually be increased depending on the radiological and clinical findings. Short leg posterior plaster splint was applied in all patients for six weeks.

Follow ups took place at 6 weeks and 3,5,7,9 and 12 months after surgery with clinical and radiological examination. Clinical evaluation included range of motion of knee and ankle, and existence of pain in adjacent joints and at the fracture site. Radiographic evaluation included the progress of the union, axial alignment. For alignment measurement full length radiograph of leg in both anteroposterior and lateral planes was taken. The length of the of the tibia was assessed clinically from medial joint line to the tip of medial malleolus and comparison was done with other leg to measure shortening. Malalignment was defined as $>5$ degree varus/valgus deformity [3]. Shortening was defined as right/left difference in the length of tibia of $>1 \mathrm{~cm}$. for measurement of deformity, the angle between distal part and proximal part was determined by measuring the angle between the line through the centre of the tibial plateu down the middle of of the proximal shaft, and the line from the centre of ankle up the middle of the distal shaft [3]. Range of motion from maximum active dorsiflexion to neutral position was determined with the help of goniometer.

\section{Bone union}

Radiologically: Bridging callus was found on was seen to cross the fracture site on three of four roentgenographic views.

Clinically: No pain or tenderness on fracture site, walking painless with full weight bearing, A classic definition of non-union was strictly followed [13]

Healing within first 6 months: Normal.

Healing within 6 to 9 months: Delayed union.

Healing more than 9 months: Non-union.

Outcome was assessed with the help of Olerud and Molander scoring system for symptoms and functional evaluation of ankle fractures at one year [14] (Table 1).

\section{Results}

Result of the present study can be summarized as:

- Mean age of patients was 41.3 years (range, 22-65) in group 1 and 39.45 years (range, $28-56)$ in group $2(\mathrm{p}=0.558)$

- $70 \%$ of the cases were males and $30 \%$ females in group 1 and $60 \%$ of cases were males and $40 \%$ were females in group 2 .

- Most common mode of trauma in both the groups is road traffic accident (10 in group 1 and 9 in group 2), followed by sports related injury in group 1 and fall from height in group 2 .

- Right side was involved in 55\% of cases in group 1 and $60 \%$ of cases in group 2. Right side was more commonly involved than left.

- Associated fibula fracture was present in $90 \%$ in group 1 and $85 \%$ patients in group 2 .

- $65 \%$ were closed and $35 \%$ grade 1 open type in group 1 cases compared to $55 \%$ closed and $45 \%$ grade 1 open in group 2 .

- Average trauma to surgery duration in group 1 was 7.4 days and in group 2 was 8.2 days which is almost equal in both groups $(\mathrm{p}=0.74)$.

- The average duration of surgery in group 2 was 90 minutes (range, 50-170 minutes) whereas average duration of surgery in group 1 was 86 minutes (range, $65-140$ minutes), $(p=0.63)$ meaning that difference between 2 groups regarding duration of surgery is not significant.

- No significant intraoperative complications were noted in both groups. Difficult reduction was observed in 3 patients in group 1 and 4 patients in group 2.

- Average time after which partial weight was started in group1 was 8 weeks (range, 6 to 11 weeks) and in group 2, 10 weeks (range, 8 to 12 weeks), $(\mathrm{p}=0.015)$ in both groups

- Average time after which patient was allowed full weight bear on the operated limb was 14.25 weeks (range,11-18 weeks) in group1 and 17.09 weeks (range, 14-21 weeks) in group2, (p = $0.001)$.

- The average time before union was 23.3 weeks (range, 16-36 weeks) in group 1 and 25.55 weeks (range, 14- 41 weeks) in group $2,(\mathrm{p}=0.299)$. Two patients in group $1(10 \%)$ failed to

\begin{tabular}{|c|c|}
\hline Rating & Results \\
\hline Excellent & $91-100 \%$ \\
\hline Good & $61-90 \%$ \\
\hline Fair & $31-60 \%$ \\
\hline Poor & $0-30 \%$ \\
\hline \multicolumn{2}{|c|}{ Table 1: Showing results based on rating. } \\
\hline
\end{tabular}


Citation: Hattarki R, Bhagat S, Patel K, Singh B, Mehendiratta D, et al. (2016) Comparative Study Between Intramedulary Interlock Nailing and Plating in Distal Metaphyseal Fractures of Tibia. Surgery Curr Res 6: 267. doi:10.4172/2161-1076.1000267

Page 5 of 7

achieve union by 24 weeks. One of them required autogenous bone grafting and went to union by 30 weeks and other patient required dynamization with bone grafting and went to union by 36 weeks.

- Shortening $(>1 \mathrm{~cm})$ was found in $25 \%$ of patients in group 1 and $10 \%$ of patients in group 2. Average shortening was $0.75 \mathrm{~cm}$ (range, $0-2$ ) in group 1 and $0.443 \mathrm{~cm}$ (range, $0-1.3$ ) in group 2 $(\mathrm{p}=0.266)$.

- Operative site infection was seen in $20 \%$ (all superficial) cases in group 1 and $35 \%$ (5 superficial and two deep) cases in group 2. Superficial infection subsided with intravenous antibiotics and continued dressing, one patient in group 2 with deep infection required wound wash with secondary suturing and healed eventually.

- Patients in group1 had screw breakage which was removed with uneventful healing, and 1 patient had screw back out which was removed percutaneously but it had no effect on healing. One case had implant failure with plate rupture in group2 due to premature weight bearing and was treated with revision plating and bone grafting.

\section{Functional outcome}

- Functional outcome was assessed by Olerud and Molander functional evaluation score at one year.

- The average Olerud and Molander score was 86 (range, 72-95) in group 1 and 78.8 (range, 59-93) in group $2(\mathrm{p}=0.008)$.

\section{Discussion}

Both groups were comparable with regards to age, sex and fracture types. Mean age of cases was 41.3 years in group 1 compared to 39.45 years in group 2. Most common mode of trauma in both groups was road traffic accident (50\% cases) followed by sports related injury in group 1 (20\% cases) and fall from height in group 2 (15\% cases).This is similar to previous study conducted by Mohammed et al. in which mean age of cases was 42 years with male to female ratio 4:1 and most common mode of trauma was road traffic accident [15] (Table 2).

Associated injuries were found in 35\% of cases in group 1 and 35\% of cases in group 2. This is due to high velocity trauma seen in motor vehicle accidents. In group 1, 2 case had chest injuries, 1 case had head injury, 3 case had fracture distal end radius and 1 case had fracture shaft radius/ulna. In group 2, 3 case had head injuries, 2 case had chest injury, 1 case with shaft femur facture and 1 with fracture mandible. Associated injuries were managed in same time the surgery was done for distal tibia. These injuries delayed the management of distal tibia fractures and in one case for delayed rehabilitation of the patient (case with shaft femur).

Associated fibula fracture was present in $90 \%$ in group 1 and $85 \%$ patients in group 2. We fixed fibula in all cases either with plating or intramedullary rod. Christopher et al. [16] reported that an intact fibula or fibular plate fixation provides initial rotational stability and minimizes varus or valgus angulation in distal tibia fractures treated with either nailing and plating group [16]. 65\% were closed and 35\% grade 1 open type in group 1 cases compared to $55 \%$ closed and $45 \%$ grade 1 open in group 2. Both groups were comparable in this regard. According to AO comprehensive classification system, there were 10 type 43A2, 5 type 43A1, 3 type 43A3, 2 type 43B1 in group 1 and 8 type 43A2, 4 type $43 \mathrm{~A} 1,5$ type 43A3,3 type 43B1 in group 2 .

The average duration of surgery in group 2 was 90 minutes (range,50-170 minutes) whereas average duration of surgery in group 1 was 86 minutes (range, $65-140$ minutes), $(p=0.63)$ meaning that

\begin{tabular}{|c|c|c|}
\hline Parameter & Degree & Score \\
\hline & None & $25 s$ \\
\hline & While walking on uneven surface & 20 \\
\hline & While walking on even surface outdoor & 10 \\
\hline & While walking indoors & 5 \\
\hline & Constant and severe & 0 \\
\hline \multirow{2}{*}{ Stiffness } & None & 10 \\
\hline & Stiffness & 0 \\
\hline \multirow{3}{*}{ Swelling } & None & 10 \\
\hline & Only Evenings & 5 \\
\hline & Constant & 0 \\
\hline \multirow{3}{*}{ Stair-climbing } & No problems & 10 \\
\hline & Impaired & 5 \\
\hline & Impossible & 0 \\
\hline \multirow{2}{*}{ Running } & Possible & 5 \\
\hline & Impossible & 0 \\
\hline \multirow{2}{*}{ Jumping } & Possible & 5 \\
\hline & Impossible & 0 \\
\hline \multirow{2}{*}{ Squatting } & No problems & 5 \\
\hline & Impossible & 0 \\
\hline \multirow{3}{*}{ Supports } & none & 10 \\
\hline & Taping, Wrapping & 5 \\
\hline & Stick or crutch & 0 \\
\hline \multirow{4}{*}{ Work, activities of daily living } & Same as before injury & 20 \\
\hline & loss of tempo & 15 \\
\hline & Change to simpler job/part time work & 10 \\
\hline & Severe impaired work capacity & 0 \\
\hline
\end{tabular}

Table 2: Comparative analysis of degree and score based on Parameter. 
difference between 2 groups regarding duration of surgery is not significant. No significant intraoperative complications were noted in both groups. Difficult reduction was observed in 3 patients in group 1 and 5 patients in group 2. Proximity to ankle joint amplifies the bending moment of the distal segment and may facilitate fracture propagation into the ankle joint $[8,9]$. No such complication was observed in our cases. Average time after which patient was allowed full weight bear on the operated limb was 14.25 weeks (range, 11-18 weeks) in group 1 and 17.09 weeks (range, 14-21 weeks) in group 2, $(\mathrm{p}=0.001)$. The cases in group 1 were able to bear weight on the operated limb in significant earlier time and able to perform independent activities at earlier time compared to group 2. There was no significant difference in union time between two groups. Higher rate of delayed union was found in group $2(25 \%)$ compared to group $1(10 \%)$. Two patients in group 1 failed to achieve union by 24 weeks. One of them required autogenous bone grafting and went to union by 34 weeks and other patient required Dynamization with bone grafting and went to union by 36 weeks. Five patients failed to achieve union by 24 weeks in group 2 (25\%). Two patients required autogenous iliac bone grafting and union was achieved by 36 weeks. One patient had implant failure with plate rupture and required revision plating with autogenous bone grafting and achieved union by 41 weeks. Angular deformity was found in 25\% of patients in group 1 and $10 \%$ of patients in group 2 . Mean angulation in group 1 was 3.4 degree (range, $0-12$ ) and 1.0 degree (range, $0-9$ ) in group $2(\mathrm{p}=0.04)$. Varus deformity of $>5$ degree was found in 4 cases and valgus deformity $>5$ degree in single case in group 1.2 cases in group 2 had deformity $>5$ degree, one had varus and other had valgus deformity. This shows that angular malalignments were more common in group 1.This complication may initially result from an incorrect entry site and entrance angle. In addition, the incidence of malalignment may be caused by instability due to the large difference between the diameter of the implant and medullary cavity of distal metaphysis. The nail does not contact the metaphyseal cortex; therefore it may translate along the interlocking screws unless anteroposterior locking is also performed [17-19]. The use of Pollar screws has been described as additional stabilization technique and reduction tool to increase the strength of bone implant construct [12].

Malalignment can lead to complaints from the patient with regard to walking, practicing sports and so forth. Puno et al. evaluated 27 patients with 28 tibial fractures at an average of 8.2 years (range, 6-12.5 years). They found a correlation joint malalignment and clinical outcome for fractures of tibia. Analysis showed that a greater degree of ankle malalignment produces poor clinical outcomes [20]. Long term effect of malalignment needs to be evaluated further. Shortening $(>1 \mathrm{~cm})$ was found in $25 \%$ of patients in group 1 and $10 \%$ of patients in group 2 . Average shortening was $0.75 \mathrm{~cm}$ (range, $0-2)$ in group 1 and $0.44 \mathrm{~cm}$ (range, $0-1.5)$ in group $2(\mathrm{p}=0.266)$. Clinically significant shortening was found in only 2 cases treated with intramedullary nailing and one case treated with plating.

Most important postoperative complication in nailing group anterior knee pain and postoperative infection patients treated with plating group. Incidence of anterior knee pain in nailing cases was $30 \%$. This complication is seen exclusively in nailing group. Operative site infection was seen in 20\% (all superficial) cases in group 1 and $35 \%$ (5 superficial and two deep) cases in group 2. Superficial infection subsided with intravenous antibiotics and continued dressing, one patient in group 2 with deep infection required wound wash with secondary suturing and healed eventually. Failure of nail or locking screws and nail is reported complication in intramedullary nailing of distal tibial fractures [21]. 2 patients in intramedullary nailing group had screw breakage which was removed with uneventful healing, and 1 patient had screw back out which was removed percutaneously but it had no effect on healing. One case had implant failure with plate rupture in group2 due to premature weight bearing and was treated with revision plating and bone grafting. Nail failure did not occur in our patients because we reamed the intramedullary canal and tried to use the nails of larger diameter. Patients treated with intramedullary nailing have statistically better ankle range of motion. Average ankle dorsiflexion was 15 degree and plantarflexion 22 degree in nailing group compared to dorsiflexion of 9 degree and plantarflexion of 13 degree in plating group. Additional procedures required in plating group were higher in plating group ( 8 case) compared to nailing group ( 5 case). 3 patients required Dynamization and 2 patients bone grafting to achieve union in nailing group. 3 patients required bone grafting, 1 case wound wash, 2 case secondary suturing and one case revision plating due to implant failure.

Higher average functional ankle score was found in nailing group compared to plating group. The average Olerud and Molander score was 86 (range, 72-95) in group 1 and 78.8 (range, 59-93) in group 2 (p $=0.008)$.

Overall results of intramedullary nailing of distal tibial metaphyseal fractures in our series were comparable to those of Robinson et al. [9], who also excluded the pilon fractures and reported that mean Olerud and Molander score was $89 \%$ in respect of prefracture level. Another study by Mosheiff et al. [22], which included seven Gustilo types 2 and 3 open fractures and used and undreamed tibial nail, contains a report that 50 of 52 distal tibial fractures united, and most of the patients regained full range of motion, although 22 patients had additional procedures, including Dynamization and bone grafting. A recent study by Dogra et al. [4], with application of shortened intramedullary nail in 15 diametaphyseal tibia fractures, also showed comparable results. All fractures united, and mean Iowa ankle score was $92 \%$ of full score. Three of their patients require additional procedures, two Dynamization cases and one nail exchange with bone grafting.

\section{Conclusion}

- Additional procedures required to achieve union were higher in locked plates.

- Use of minimally invasive technique in plating reduces the wound problems and successively employed with bridge plating of communited metaphyseal fractures of distal tibia.

- Functional ankle scores were higher in nailing group compared to plating group.

- Anterior knee pain is noted only with intramedullary technique and no knee complications were noted with plating group.

- Intramedullary nail being load sharing device, comparatively early mobilization can be started. Prolonged duration of protected weight bearing was required in patients treated with locked plate and screws.

\section{References}

1. Fan CY, Chiang CC, Chuang TY, Chiu FY, Chen TH (2005) Interlocking nails for displaced metaphyseal fractures of distal tibia. Injury, 36: 669-674.

2. Ovadia DN, Beals RK (1986) Fractures of tibial plafond. J Bone Joint Surg Am 68: $543-551$

3. Robinson CM, McLauchlan GJ, Mc Lean IP, Court-Brown CM (1995) Dista metaphyseal fractures of tibia with minimal involvement of ankle: classification and treatment by locked intramedullary nailing. J Bone Joint Surg $\mathrm{Br}$ 77: 781-787.

4. Dogra AS, Ruitz al, Thompson NS, Nolan PC et al. (2000) Dia-metaphysea 
Citation: Hattarki R, Bhagat S, Patel K, Singh B, Mehendiratta D, et al. (2016) Comparative Study Between Intramedulary Interlock Nailing and Plating in Distal Metaphyseal Fractures of Tibia. Surgery Curr Res 6: 267. doi:10.4172/2161-1076.1000267

distal tibial fractures: treatment with shortened intramedullary nail; a review of 15 cases. Injury 31: 799-804.

5. Im GI, Tae SK (2005) Distal metaphyseal fractures of tibia: a prospective randomized trial of closed reduction and intramedullary nail versus open reduction and plate and screws fixation. J Trauma 59: 1219-1223.

6. Kuhn S, Hansen M, Rommens PM (2007) Extending the indication intramedullary nailing of tibial fractures. Eur J Trauma Emerg Surg 33: 159-169.

7. Russo CR, Lauretani F, Seeman E, Bartali B, Bandinelli S, et al. (2006) Structural adaptations to bone loss in ageing man and women. Bone 38: 112-118.

8. Steinberg EL, Geller DS, Yacoubian SV, Shasha N, Dekel S, et al. (2006) Intramedullary fixation of tibial shaft fractures using an expandable nail ; an early results of 54 acute tibial shaft fractures. J Orthop Trauma 20: 303-309.

9. Rockwood and Green's, fractures in adults 7th edition, volume 2 . Fractures of tibia and fibula, 1884-1887.

10. Borelli J Jr, Prickett W, Song E, Becker D, Ricci W (2002). Extraosseous blood supply of tibia and the effect of different plating techniques: a human cadaveric study. J Orthop Trauma 16: 691-695.

11. Gustilo RB, Anderson JT (1976) Prevention of infection in the treatment of 1025 open fractures of long bones: retrospective and prospective analysis. J Bone $\mathrm{J}$ Surg Am 58: 453-458.

12. Krettek C, Miclau T, Schandelmaier P, Stephan C, Möhlmann U, et al. (1999) The mechanical effect of blocking screws (Pollar screws)in stabilizing tibia fracture with short proximal or distal fragments after insertion of small diameter intramedullary nails. J Orthop Trauma12: 550-553.
13. Ronga M, longo U, Maffuli N (2010) Minimally invasive locked plating is safe and effective. Clin Orthop Relat Res 468: 975-982.

14. Olerud $\mathrm{C}$, Molande $\mathrm{H}$ (1984) A scoring scale for evaluation after ankle fractures. Arch Orthop Trauma Surg 103: 190-194.

15. Mohammed A, Sarawan R, Zammit J, King R (2008) Intramedullary nailing in distal third tibial fractures: distal locking screws and fracture nonunion. Int Orthop 32: 547-549.

16. Stewart CM, Kiner D, Nowotarski P (2013) Intramedullary Nail Fixation of Fibular Fractures Associated With Tibial Shaft and Pilon Fractures. J Orthop Trauma 27: 114-117.

17. Freedmen EL, Johnson EE (1995) Radiographic analysis of tibial fracture malalignment following intramedullary nailing. Clin Orthop Relat Res 315: 2533.

18. Henley BM, Meier T, Tencer AF (1993) Inflences of some design parameters on biomechanics of the unreamed tibial intramedullary nail. J Ortho Trauma 7: 311-319.

19. Watson JT (1994) Treatment of the unstable fractures of shaft of tibia. J Bone Joint Surg Am 76: 1575-1584.

20. Puno RM, Vaughan JJ, Stetten ML, Johnson JR (1991) Long term effects of angular malunion on the knee and ankle joints. $J$ of Orthop Trauma 3: 247-254.

21. Boenisch UW, de Boer PG, Journaeux SF (1996) Unreamed intramedullary tibial nailing-fatigue of locking bolts. Injury 27: 265-267.

22. Mosheiff R, Safran O, Segal D, Liebergall M (1999) The undreamed tibial nail in the treatment of distal metaphyseal fractures. Injury 30: 83-90. 